\title{
artigo
}

Silva, K.A.; Abreu, S.M.; Fernandes, V.C.; Nassar, P.R.B.; Souza, R.R.; Marta, C.B.;

Diagnósticos de enfermagem em gestante no serviço de atendimento movél de urgência

\section{Diagnósticos de enfermagem em gestante no serviço de atendimento movél de urgência}

\begin{abstract}
RESUMO
Objetivo: Identificar quais são os diagnósticos enfermagem, dentro do atendimento pré-hospitalar móvel de urgência. Método: Trata-se de um estudo bibliográfico, descritivo, do tipo revisão integrativa, de 2011 a 2018, com busca de artigos nas bases de dados LILACS, MEDLINE, BDENF e Biblioteca Virtual. Apresentam-se os resultados na tabela, em seguida, discutidos com a literatura. Resultados: Selecionaram-se sete artigos referentes aos diagnósticos de enfermagem (NANDA-1), que no trauma em gestante esta assistência prestada seja importante e benéfica para que os cuidados sejam de acordo com as necessidades das mulheres que vivenciam a parturição em um ambiente de atendimento móvel de urgência (SAMU). Conclusão: Este estudo identificou diagnósticos de enfermagem de trauma em mulheres grávidas para que os profissionais de saúde proporcionem um melhor atendimento no ambiente pré-hospitalar. Portanto entende-se, que este processo deve ser adotado em todos os serviços deste cenário.
\end{abstract}

DESCRITORES: Diagnostico de Enfermagem; Gestantes; Serviços Médicos de Emergência.

\section{ABSTRACT}

Objective: Identify the nursing diagnoses within the emergency mobile prehospital care. Method: This is a descriptive bibliographic study, integrative review, from 2011 to 2018, searching for articles in the LILACS, MEDLINE, BDENF and Virtual Library databases. The results are presented in the table, then discussed with the literature. Results: We selected seven articles referring to nursing diagnoses (NANDA-1), which in trauma in pregnant women this care provided is important and beneficial so that care is in accordance with the needs of women who experience parturition in an environment of emergency mobile service (SAMU). Conclusion: This study identified nursing diagnoses of trauma in pregnant women for health professionals to provide better care in the prehospital setting. Therefore, it is understood that this process should be adopted in all services of this scenario.

KEYWORDS: Nursing Diagnosis; Pregnant; Emergency Medical Services.

\section{RESUMEN}

Objetivo: identificar los diagnósticos de enfermería dentro de la atención prehospitalaria móvil de emergencia. Método: Este es un estudio bibliográfico descriptivo, revisión integradora, de 2011 a 2018, que busca artículos en las bases de datos LILACS, MEDLINE, BDENF y Biblioteca Virtual. Los resultados se presentan en la tabla, luego se discuten con la literatura. Resultados: Seleccionamos siete artículos referidos a diagnósticos de enfermería (NANDA-1), que en trauma en mujeres embarazadas, esta atención brindada es importante y beneficiosa para que la atención esté de acuerdo con las necesidades de las mujeres que experimentan el parto en un entorno de servicio móvil de emergencia (SAMU). Conclusión: Este estudio identificó diagnósticos de trauma de enfermería en mujeres embarazadas para que los profesionales de la salud brinden una mejor atención en el entorno prehospitalario. Por lo tanto, se entiende que este proceso debe adoptarse en todos los servicios de este escenario.

PALABRAS CLAVE: Diagnóstico de Enfermería; Embarazadas; Servicios Médicos de Emergencia.

RECEBIDO EM: 11/11/2019 APROVADO EM: 12/11/2019

\section{Karen Albuquerque da Silva}

Pós-graduação de Enfermagem em Alta Complexidade pela Universidade Veiga de Almeida.

\section{Sabrina Marins Abreu}

Pós-graduação de Enfermagem em Alta Complexidade pela Universidade Veiga de Almeida. ENFERMEIRA / PAISMCA -Secretaria Municipal de saúde do município de Maricá.

\section{Vladimir Chaves Fernandes}

Mestre em Enfermagem - EEAN UFRJ. Especialista em Gestão de Emergências em Saúde Pública - Instituto Sírio Libanês.

Oficial Enfermeiro CBMERJ Prof. Universidade Veiga de Almeida. 


\section{Pedro Ruiz Barbosa Nassar}

Doutor em ciências pela Unirio. Mestre em enfermagem pela Unirio. Especialista em gestão dos serviços de saúde e enfermagem pela UFF. Prof. Adjunto do departamento de fundamentos de enfermagem e administração. EEAAC/UFF.

\section{Rodrigo Rocha de Souza}

Enfermeiro Obstétrico. Coordenador da pós-graduação em Enfermagem obstétrica da Universidade Veiga de Almeida.

\section{Frances Tavares}

Enfermeira especialista em Enfermagem em Alta Complexidade pela Universidade Veiga de Almeida.

\section{Cristiano Bertolossi Marta}

Enfermeiro. Pós-doutorado pela Escola de Enfermagem Aurora Afonso Costa da Universidade Federal Fluminense. Professor Adjunto do Departamento de Fundamentos de Enfermagem da Faculdade de Enfermagem da Universidade do Estado do Rio de Janeiro. Diretor de pesquisa e Coordenador da pós-graduação de Enfermagem em Alta Complexidade da Universidade Veiga de Almeida. Pesquisador líder do núcleo de Avaliação de Tecnologias e Economia em Saúde e Segurança do Paciente (NATESSP) da Universidade Veiga de Almeida.

\section{INTRODUÇÃO}

0 Serviço de Atendimento Móvel de Urgência (SAMU) é o principal componente da Política Nacional de Atenção às Urgências que tem como finalidade proteger a vida das pessoas e garantir a qualidade no atendimento no Sistema Único de Saúde, de acordo com seus princípios doutrinários, a universalidade, a equidade e a integralidade ${ }^{(1)}$.

Destaca-se que, no Brasil, a implantação dos serviços de atendimento pré-hospitalar ocorreu no início da década de 90 , o qual passou a ser denominado de Serviço de Atenção Móvel de Urgência (SAMU), a partir do Plano Nacional de Atendimento à Urgência e Emergência em $2003^{(2)}$.

Acrescenta-se que, o pré-atendimento móvel ficou também sob a responsabilidade do Serviço de Atendimento Móvel de Urgência (SAMU), regulamentado pela Portaria n. ${ }^{\circ}$ 1.864/GM. Ressalta-se que tem, como função, o salvamento e o resgate realizados nas residências, locais de trabalho e vias públicas em todo o território nacional por meio do acesso pela Central de Regulação n. ${ }^{\circ} 192^{(3)}$.

Evidencia-se que o SAMU tem, como principal foco, o atendimento em situações de urgência e emergência a usuários com demandas clínica, pediátrica, psiquiátrica, cirúrgica e gineco-obstétrica. Mostra-se, nessa última área, fundamental no atendimento e no transporte rápido de gestantes em trabalho de parto, nos quais há risco de morte para a mãe e/ou feto, ou seja, é uma importante ferramenta para reduzir o número de mortes em função do retardo ao acesso e minimizar sequelas decorrentes do atendimento tardio ${ }^{(4)}$.

No período gravídico-puerperal, o atendimento multiprofissional é importante, pois a maioria necessita de um mínimo de intervenções. Em algumas situações podem ocorrer complicações ou emergências com risco de vida, necessitando ser identificadas com eficiência. Portanto, a assistência deve envolver ações efetivas desde os profissionais até o gestor municipal para garantir uma assistência materno-fetal de qualidade e sem riscos ${ }^{(5)}$.

A equipe de saúde deve estar capacitada técnica e cientificamente, buscando aprimorar seus conhecimentos para atuar diretamente, proporcionando uma boa assistência integral para diminuir os riscos de morbimortalidade materna e neonatal, apresentando medidas preventivas e reparadoras a essas gestantes vítimas de trauma que tenham risco eminente de morte.

A padronização da assistência voltada às vítimas de trauma vem sendo cada vez mais implementada pelas instituições de saúde no Brasil, no entanto, há uma necessidade de personalizar esse atendimento, devido às diferentes formas de apresentação, gravidade e complexidade em particular de cada um desses pacientes. Nesse sentido, a utilização de diagnósticos de enfermagem mostra-se como a melhor e mais prática solução para atuação da enfermagem no cenário da emergência ${ }^{(6)}$.

$\mathrm{O}$ estabelecimento de diagnósticos de enfermagem é ação privativa do enfermeiro e permite, em sua elaboração, a composição de uma ampla rede documental de informações codificadas, de conceitos e evidências científicas que, certamente, contribuem para o delineamento de conhecimentos da profissão, sobretudo, na escolha de intervenções e resultados esperados ${ }^{(7)}$.

Portanto, os diagnósticos de enfermagem proporcionam a base para a seleção de intervenções de enfermagem para atingir resultados pelos quais a enfermagem é responsável, sobretudo, junto a vítimas graves e de maior complexidade ${ }^{(8,9)}$.

Desta forma, o problema de pesquisa a ser estudado é: Quais são os diagnósticos de enfermagem no trauma em gestante encontrados no serviço de atendimento móvel de urgência? Para responder a essa pergunta, definimos como objetivo identificar quais são os diagnósticos enfermagem, dentro do atendimento pré-hospitalar móvel de urgência no Brasil, que possam trazer benefícios para uma sobrevida melhor do binômio mãe-bebê, proporcionando uma boa assistência dos profissionais de saúde.

A justificativa desta pesquisa em relação ao atendimento assistencial dos profissionais de saúde é que existe um papel fundamental na construção deste vínculo com o cuidado integral às gestantes vítimas de traumas na emergência, com a finalidade de diminuir esses riscos de morbimortalidade que acometem estas mulheres, proporcionando um bom diagnóstico de enfermagem.

Evidenciando esses fatos, espera-se que 


\section{artigo}

Silva, K.A.; Abreu, S.M.; Fernandes, V.C.; Nassar, P.R.B.; Souza, R.R.; Marta, C.B.;

Diagnósticos de enfermagem em gestante no serviço de atendimento movél de urgência

seja um assunto relevante a nível assistencial, cada dia mais no serviço, para que ocorram mudanças significativas em prol da saúde do cliente.

\section{METODOLOGIA}

$\mathrm{O}$ artigo desenvolvido foi com base em uma pesquisa de revisão integrativa, de natureza qualitativa e descritiva, buscando discutir com a utilização do diagnóstico e intervenção de enfermagem no trauma, proporcionado uma melhor assistência no atendimento a gestante. A pesquisa foi realizada entre março a outubro 2019, pelo portal Biblioteca Virtual em Saúde (BVS) com base em levantamentos de documentos indexados nas bases de dados da LI-

\section{Fluxograma 1. Seleção dos estudos. Rio de Janeiro, RJ, Brasil, 2019}
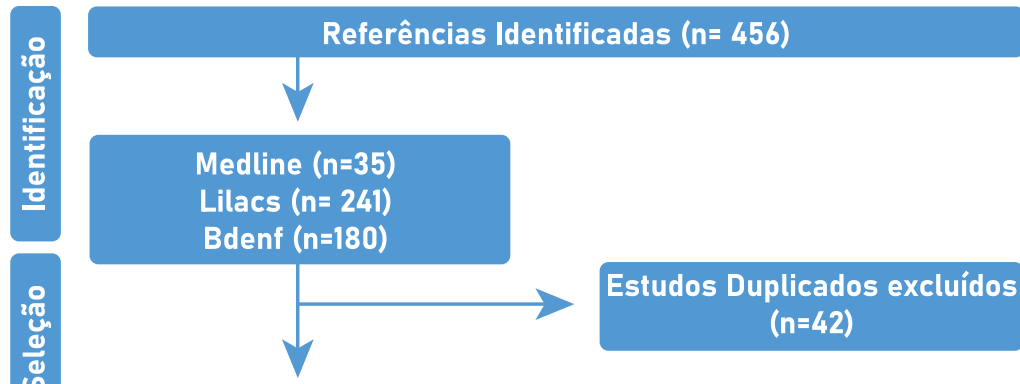

Referências selecionadas para leitura $(n=30)$
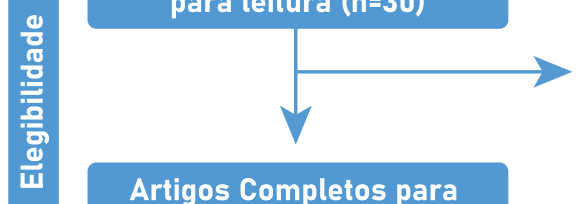

Artigos Completos excluídos $(n=23)$
Artigos Completos para

Avaliaçäo $(n=7)$
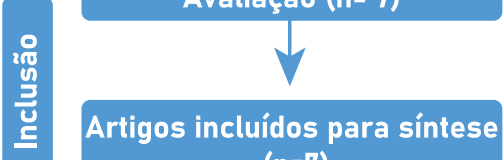

Artigos incluídos para sintese

$$
(n=7)
$$

\begin{tabular}{|c|c|c|c|c|c|}
\hline No & TÍTULO & AUTOR & $\begin{array}{l}\text { ANO/ } \\
\text { PAIIS }\end{array}$ & RESULTADOS & CONCLUSÃO \\
\hline 1 & $\begin{array}{l}\text { Diagnósticos e } \\
\text { intervenções de } \\
\text { enfermagem em } \\
\text { vítimas de trauma } \\
\text { durante atendimento } \\
\text { pré-hospitalar } \\
\text { utilizando a CIPE }\end{array}$ & $\begin{array}{l}\text { Thaís Honório } \\
\text { Linsl; Ana Xênia } \\
\text { Buarque Coelho } \\
\text { de Limall; } \\
\text { Regina Célia } \\
\text { Sales Santos } \\
\text { Veríssimolll; } \\
\text { Janine Melo de } \\
\text { OliveiralV }\end{array}$ & $\begin{array}{l}\text { 2013/ } \\
\text { Brasil }\end{array}$ & $\begin{array}{l}\text { Foram formulados } 33 \text { diagnósticos } \\
\text { e } 14 \text { intervenções. Destacam-se } \\
\text { os diagnósticos "ferida por arma de } \\
\text { fogo atual" e "sistema cardiovascular } \\
\text { e respiratório comprometido" } \\
\text { por evidenciarem a natureza dos } \\
\text { agravos atendidos pelo serviço. } \\
\text { Entre as intervenções sobressaem- } \\
\text { se: "implementar oxigenoterapia por } \\
\text { máscara de oxigênio" e "restabelecer } \\
\text { sistema cardiovascular e respiratório } \\
\text { com manobras de ressuscitação". }\end{array}$ & $\begin{array}{c}\text { Conclui-se que a identificação } \\
\text { de diagnósticos e intervenções } \\
\text { de enfermagem CIPE }{ }^{\circledR} \text { mais } \\
\text { frequentes em vítimas de } \\
\text { trauma durante atendimento } \\
\text { pré-hospitalar pode contribuir } \\
\text { em cuidados mais direcionados } \\
\text { e sistematizados, além de } \\
\text { favorecer a documentação de } \\
\text { enfermagem. }\end{array}$ \\
\hline
\end{tabular}

LACS, MEDLINE e BDENF, através dos Descritores em Ciências da Saúde (DeCS) "Diagnostico de Enfermagem", "Gestantes" e "Serviços Médicos de Emergência”.

Utilizou-se como questão pesquisa deste estudo: Quais são os diagnósticos de enfermagem a gestante no trauma em um serviço de atendimento de urgência?

Elencaram-se, como critérios de inclusão do estudo, artigos científicos em uma visão temporal entre 2011 a 2018, em português e disponíveis em texto completo. Obtendo-se uma amostra de 20 artigos e, após a leitura extensiva, 07 responderam aos objetivos e aos critérios de inclusão do estudo por análise temática. Para os critérios de exclusão, foram retiradas as dissertações, teses, resumos, artigos repetidos e aqueles que não respondiam à questão norteadora do estudo.

\section{RESULTADOS}

Dos 07 artigos que compóem a amostra, dois (02) estão disponíveis na MEDLINE, três (03) na LILACS e dois (02) na BDENF. Em relação ao ano de publicação, as mesmas ocorreram entre 2009 a 2016, sendo que os anos de 2013 e 2016 se destacaram nas publicações. Quanto ao idioma, os 07 artigos foram editados em português. No tocante ao país de realização da pesquisa, todos os estudos foram realizados no Brasil, como mostrado no Quadro 1.

\section{Quadro 1. Informação dos artigos selecionados. Rio de Janeiro, RJ, Brasil, 2019}




\begin{tabular}{|c|c|c|c|c|c|}
\hline 2 & $\begin{array}{l}\text { Diagnósticos de } \\
\text { enfermagem em } \\
\text { vítimas de trauma } \\
\text { atendidas em um } \\
\text { serviço pré-hospitalar } \\
\text { avançado móvel }\end{array}$ & $\begin{array}{l}\text { Cyrillo, Regilene } \\
\text { Molina Zacareli; } \\
\text { Dalri, Maria } \\
\text { Célia Barcellos; } \\
\text { Canini, Silvia } \\
\text { Rita Marin da } \\
\text { Silva; Carvalho, } \\
\text { Emilia Campos } \\
\text { de; Lourencini, } \\
\text { Renata Roque. }\end{array}$ & $\begin{array}{l}\text { 2009/ } \\
\text { Brasil }\end{array}$ & $\begin{array}{l}\text { Foi elaborado e validado um instrumento } \\
\text { de coleta de dados, segundo o modelo } \\
\text { conceitual de Horta; os dados coletados } \\
\text { foram validados por peritos na temática. } \\
\text { O projeto foi aprovado por Comitê de Ética } \\
\text { e Pesquisa. Foram avaliadas } 23 \text { vítimas } \\
\text { de trauma, sendo } 17(73,9 \%) \text { homens, } 16 \\
(69,5 \%) \text { com idade entre } 18 \text { e } 30 \text { anos e } 14 \\
(60,1 \%) \text { vítimas de acidentes de transito. } \\
\text { Os diagnósticos de enfermagem mais } \\
\text { frequentes foram: Risco para infecção } \\
(91 \%) \text {, Risco para trauma (82\%), Dor aguda } \\
(74 \%) \text {, Integridade tissular prejudicada (65\%), } \\
\text { Volume de líquidos deficiente (43\%) e Risco } \\
\text { para volume de líquido deficiente (43\%). }\end{array}$ & $\begin{array}{l}\text { Este estudo permitiu identificar } \\
\text { os principais diagnósticos de } \\
\text { enfermagem nesta clientela } \\
\text { específica, os quais poderão } \\
\text { fornecer importantes subsídios } \\
\text { para futuras investigações, } \\
\text { sobretudo abordando } \\
\text { intervenções de enfermagem. }\end{array}$ \\
\hline 3 & $\begin{array}{l}\text { Ocorrências obsté- } \\
\text { tricas atendidas pelo } \\
\text { Serviço de Atendimen- } \\
\text { to Móvel de Urgência }\end{array}$ & $\begin{array}{l}\text { Jéssica Gomes } \\
\text { da Silva1, Suzel } \\
\text { Regina Ribeiro } \\
\text { Chavaglia2, Ma- } \\
\text { riana Torreglosa } \\
\text { Ruiz3 }\end{array}$ & $\begin{array}{l}\text { 2016/ } \\
\text { Brasil }\end{array}$ & $\begin{array}{l}\text { Informa-se que os chamados por causas } \\
\text { obstétricas representaram 0,40\% dos } \\
\text { atendimentos do SAMU; a idade média } \\
\text { das gestantes foi de } 25,08 \text { anos; a maioria } \\
\text { era primigesta, estava no terceiro trimes- } \\
\text { tre gestacional, realizou pré-natal e as } \\
\text { principais queixas foram relacionadas ao } \\
\text { trabalho de parto }(91,3 \%) \text {. Houve signifi- } \\
\text { cância estatística entre o acionamento do } \\
\text { SAMU por queixas referentes ao trabalho } \\
\text { de parto e o terceiro trimestre gestacional } \\
\text { indicando a pertinência dos chamados. }\end{array}$ & $\begin{array}{c}\text { Conclui-se, que ao conhecer as } \\
\text { necessidades das mulheres que } \\
\text { buscam atendimento no serviço } \\
\text { pré-hospitalar e traçar o perfil } \\
\text { de atendimentos são informa- } \\
\text { ções essenciais para subsidiar } \\
\text { as ações e as políticas públicas } \\
\text { que possibilitem oferecer as- } \\
\text { sistência de qualidade e reduzir } \\
\text { a morbimortalidade materna e } \\
\text { neonatal.(AU) }\end{array}$ \\
\hline 4 & $\begin{array}{l}\text { Diagnósticos reais e } \\
\text { proposta de interven- } \\
\text { ções de enfermagem } \\
\text { para os pacientes } \\
\text { vítimas de múltiplos } \\
\text { traumas }\end{array}$ & $\begin{array}{l}\text { Kátia Cilene } \\
\text { Godinho Ber- } \\
\text { toncellol; Cibele } \\
\text { D'Avila Kramer } \\
\text { Cavalcantill; } \\
\text { Patrícia Ilhalll }\end{array}$ & $\begin{array}{l}\text { 2013/ } \\
\text { Brasil }\end{array}$ & $\begin{array}{c}\text { Foram avaliados } 41 \text { pacientes encami- } \\
\text { nhados por serviços de atendimento } \\
\text { pré-hospitalar, maiores de } 18 \text { anos, } \\
\text { de ambos os sexos e não gestantes } \\
\text { utilizando como referencial teórico a } \\
\text { Taxonomia Il da NANDA Internacional e } \\
\text { a Nursing Interventions Classification. } \\
\text { Foram identificados } 25 \text { diagnósticos. } \\
\text { Os mais prevalentes foram: dor aguda; } \\
\text { mobilidade física prejudicada; deambu- } \\
\text { lação prejudicada; déficit no autocuidado } \\
\text { para banho; déficit no autocuidado } \\
\text { para vestir-se; déficit no autocuidado } \\
\text { para higiene íntima; mobilidade no leito } \\
\text { prejudicada; capacidade de transferência } \\
\text { prejudicada; integridade da pele preju- } \\
\text { dicada; integridade tissular prejudicada; } \\
\text { déficit no autocuidado para alimentação; } \\
\text { padrão respiratório ineficaz. }\end{array}$ & $\begin{array}{l}\text { Após, foram propostas } 82 \\
\text { intervenções de enfermagem } \\
\text { que pudessem propiciar uma } \\
\text { redução de danos a esses } \\
\text { pacientes. }\end{array}$ \\
\hline 5 & $\begin{array}{l}\text { Avaliando diagnósticos } \\
\text { e intervençães de en- } \\
\text { fermagem no trabalho } \\
\text { de parto e na gestação } \\
\text { de risco }\end{array}$ & $\begin{array}{l}\text { Ana Lúcia de } \\
\text { Medeirosa ; Sér- } \\
\text { gio Ribeiro dos } \\
\text { Santosa; Rô- } \\
\text { mulo Wanderley } \\
\text { de Lima Cabralb } \\
\text {; Juliana Paiva } \\
\text { Góes Silvac; } \\
\text { Neyce de Matos } \\
\text { Nascimentoc }\end{array}$ & $\begin{array}{l}\text { 2016/ } \\
\text { Brasil }\end{array}$ & $\begin{array}{c}\text { Os diagnósticos mais evidenciados } \\
\text { no trabalho de parto: dor aguda }(62 \%) \text {, } \\
\text { fadiga }(24,7 \%) \text { e ansiedade }(22 \%) \text {. Para as } \\
\text { gestantes de alto risco: sono e repouso } \\
\text { prejudicados }(100 \%) \text {, risco de infecção } \\
(81,8) \text { e ansiedade }(77,2 \%) \text {. As inter- } \\
\text { venções foram: lavar as mãos }(80,8 \%), \\
\text { identificar e acomodar no leito }(78 \%) \text {. }\end{array}$ & $\begin{array}{l}\text { Os diagnósticos expressam as } \\
\text { necessidades na parturição e } \\
\text { nas alterações psicobiológicas } \\
\text { na gestação de risco. As inter- } \\
\text { venções estão desarticuladas } \\
\text { dos diagnósticos, necessitan- } \\
\text { do de revisões e mudanças. }\end{array}$ \\
\hline
\end{tabular}




\section{artigo}

Silva, K.A.; Abreu, S.M.; Fernandes, V.C.; Nassar, P.R.B.; Souza, R.R.; Marta, C.B.;

Diagnósticos de enfermagem em gestante no serviço de atendimento movél de urgência

\begin{tabular}{|c|c|c|c|c|c|}
\hline 6 & $\begin{array}{l}\text { Diagnósticos de enfer- } \\
\text { magem em vítimas de } \\
\text { trauma nas primeiras } \\
\text { seis horas após o } \\
\text { evento }\end{array}$ & $\begin{array}{l}\text { Sallum, Ana Ma- } \\
\text { ria Calil; Sousa, } \\
\text { Regina Marcia } \\
\text { Cardoso de }\end{array}$ & $\begin{array}{l}\text { 2012/ } \\
\text { Brasil }\end{array}$ & $\begin{array}{l}\text { Os diagnósticos de enfermagem mais } \\
\text { frequentes foram: Risco de Infecção } \\
(84,5 \%) \text {, Integridade da pele prejudicada } \\
(77,9 \%) \text {, Dor aguda }(71,5 \%) \text {, Conforto } \\
\text { prejudicado }(68,3 \%) \text { e Integridade tis- } \\
\text { sular prejudicada }(54,1 \%) \text {. A associação } \\
\text { entre diagnósticos de enfermagem e } \\
\text { mortalidade foi observada em } 28 \text { ( } 66,7 \%) \\
\text { dos diagnósticos identificados. }\end{array}$ & $\begin{array}{l}\text { Os dados acrescentaram } \\
\text { informações que poderão } \\
\text { auxiliar na formação e atuação } \\
\text { do enfermeiro no cenário das } \\
\text { emergências em trauma e } \\
\text { evidenciaram o potencial dos } \\
\text { diagnósticos de enfermagem } \\
\text { para avaliar os resultados e a } \\
\text { qualidade da assistência. }\end{array}$ \\
\hline 7 & $\begin{array}{l}\text { Diagnósticos de enfer- } \\
\text { magem em pacientes } \\
\text { numa unidade de } \\
\text { emergência }\end{array}$ & $\begin{array}{l}\text { Salgado, Patrícia } \\
\text { Oliveira; Gon- } \\
\text { çale, Paula Ca- } \\
\text { roline; Dantas, } \\
\text { Raquel Batista; } \\
\text { Castro, Marcelo } \\
\text { Augusto; Chian- } \\
\text { ca, TâniaCouto } \\
\text { Machado. }\end{array}$ & $\begin{array}{l}\text { 2013/ } \\
\text { Brasil }\end{array}$ & $\begin{array}{l}\text { após o processo de exclusão das repeti- } \\
\text { ções obteve-se } 67 \text { títulos diagnósticos } \\
\text { de enfermagem, } 25 \text { títulos DE foram } \\
\text { formulados para } 10 \% \text { ou mais dos pa- } \\
\text { cientes, sendo que dois desses (Nutrição } \\
\text { desequilibrada: menos do que as neces- } \\
\text { sidades corporais e Risco de integridade } \\
\text { da pele prejudicada) apresentaram } \\
\text { frequência maior ou igual a } 50 \% \text {. Dos } 13 \\
\text { domínios da Taxonomia II da NANDA-I } \\
10 \text { foram identificados entre os } 67 \text { títu- } \\
\text { los elaborados. }\end{array}$ & $\begin{array}{c}\text { Verifica-se a necessidade } \\
\text { de pesquisas relacionadas à } \\
\text { identificação dos diagnósticos } \\
\text { de enfermagem nos serviços } \\
\text { de urgência e emergência. } \\
\text { (AU) }\end{array}$ \\
\hline
\end{tabular}

\section{DISCUSSÃO}

As mulheres passam, em seu ciclo vital, por modificações provocadas pelas suas conformações biológicas que influenciam na sua trajetória sociopsicobiológica, limitando-as, muitas vezes, a exercer papéis já estabelecidos pelas sociedades, isto é, cada fase requer uma identidade que tem influência direta na qualidade de vida ${ }^{(10)}$.

Os estudos encontrados indicaram os diagnósticos na gestante dentro trauma, mas antes vale ressaltar a importância na qual a gravidez se constitui em um fenômeno fisiológico que se caracteriza por modificações e adaptações biológicas e psicossociais, que geralmente evolui de forma saudável. Contudo, em uma parcela desta população, por fatores ou características específicas, algumas gestantes evoluem com intercorrências e/ou agravos, ao que se classifica como gravidez de alto risco, o que poderá gerar uma evolução desfavorável ao binômio mãe/filho ${ }^{(10)}$.

Diante do pressuposto, é importante organizar a assistência de enfermagem, tanto ao método quanto ao trabalho e dar maior enfoque científico à profissão. O Conselho Federal de Enfermagem (COFEN), em Resolução n. ${ }^{\circ} 358$, considera que a Sistematização da Assistência de Enfermagem (SAE), enquanto estratégia para o alcance de padronização e qualidade deva ser implantada, organiza o trabalho profissional quanto ao método científico adotado, pessoal e instrumentos necessários para sua realização, além de garantir um cuidado humanizado, contínuo e de qualidade ${ }^{(11)}$.

Associado a isso, o processo de enfermagem desenvolve-se em um processo de cinco fases sequenciais e inter-relacionadas (histórico, diagnóstico, planejamento, implemen- tação e avaliação), coerentes com a evolução da profissão e que, quando levado para o $\mathrm{APH}$, molda-se à biomecânica e ao $\mathrm{ABC}$ $\mathrm{DE}$ do trauma podendo então ser aplicado em um serviço de atendimento móvel de urgência ${ }^{(12,13)}$, como os diagnósticos de enfermagem que proporcionam a sustentação para a seleção de intervenções de enfermagem, a fim de atingir resultados pelos quais a enfermagem é responsável ${ }^{(14,15)}$.

Identifica-se no Quadro 2 os diagnósticos de enfermagem encontrados dentro do trauma, segundo os domínios da classificação da (NANDA-I). Durante a investigação, dentro dos estudos, foram encontrados 11 diagnósticos reais de enfermagem.

Os títulos diagnósticos foram descritos conforme a publicação brasileira de 20182020 da classificação de diagnósticos de enfermagem da NANDA-I: Definições e classificação(16). $^{(16)}$.

Quadro 2: Diagnósticos de Enfermagem (NANDA 2018-2020): Definições e classificação. Rio de Janeiro, RJ, Brasil, 2019

\begin{tabular}{|l|l|l|}
\hline \multicolumn{1}{|c|}{ DIAGNÓSTICOS DE ENFERMAGEM } & \multicolumn{1}{|c|}{ CARACTERÍSTICAS DEFINIDORAS } & \multicolumn{1}{c|}{ FATOR RELACIONADO } \\
\hline Risco de Infecção & $\begin{array}{l}\text { Suscetibilidade a invasão e multiplicação } \\
\text { de organismos patogênicos que pode } \\
\text { comprometer a saúde. }\end{array}$ & $\begin{array}{l}\text { Imunossupressão; Leucopenia; Resposta } \\
\text { inflamatória suprimida; Ruptura prematura } \\
\text { de membrana amniótica; Ruptura } \\
\text { prolongada de membrana amniótica, entre } \\
\text { outros }\end{array}$ \\
\hline
\end{tabular}




\begin{tabular}{|c|c|c|}
\hline Risco de Sangramento & $\begin{array}{l}\text { Suscetibilidade à redução no volume de sangue que } \\
\text { pode comprometer a saúde. }\end{array}$ & $\begin{array}{l}\text { Trauma, coagulopatia inerente, } \\
\text { coagulação intravascular disseminada, } \\
\text { complicação gestacional, entre outros. }\end{array}$ \\
\hline Risco de Choque & $\begin{array}{l}\text { Suscetibilidade a fluxo sanguíneo inadequado para } \\
\text { os tecidos do corpo, que pode levar a disfunção } \\
\text { celular que ameaça a vida que pode comprometer } \\
\text { a saúde. }\end{array}$ & $\begin{array}{l}\text { Hipotensão, Hipovolemia; Hipoxemia, } \\
\text { infecção, sepse, entre outros. }\end{array}$ \\
\hline Dor Aguda & $\begin{array}{l}\text { Experiência sensorial e emocional desagradável } \\
\text { associada à lesão tissular real ou potencial, ou } \\
\text { descrita em termos de tal lesão (International } \\
\text { Association dor the Study of Pain); início súbito ou } \\
\text { lento de intensidade leve a intensa, com término } \\
\text { antecipado ou previsivel e com duração menor que } \\
3 \text { meses }\end{array}$ & $\begin{array}{l}\text { Agente físico lesivo, Agente químico } \\
\text { lesivo. }\end{array}$ \\
\hline Ansiedade & $\begin{array}{l}\text { Sentimento vago e incômodo de desconforto ou } \\
\text { temor, acompanhado por resposta autonômica } \\
\text { (a fonte é frequentemente não específica ou } \\
\text { desconhecida para o indivíduo); sentimento de } \\
\text { apreensão causado pela antecipação de perigo. } \\
\text { É um sinal de alerta que chama a atenção para } \\
\text { um perigo iminente e permite ao indivíduo tomar } \\
\text { medidas para lidar com a ameaça }\end{array}$ & $\begin{array}{l}\text { Ameaça à condição atual. Ameaça de } \\
\text { morte, estressores, necessidades não } \\
\text { atendidas, entre outros. }\end{array}$ \\
\hline Risco de Integridade da pele prejudicada & $\begin{array}{l}\text { Suscetibilidade a alteração na epiderme e/ou derme } \\
\text { que pode comprometer a saúde. }\end{array}$ & Extremo de idade, entre outros. \\
\hline Mobilidade física prejudicada & $\begin{array}{l}\text { Limitação no movimento independente e voluntário } \\
\text { do corpo ou de uma ou mais extremidades. }\end{array}$ & $\begin{array}{l}\text { Ansiedade, dor, controle muscular } \\
\text { diminuído, rigidez articular, entre outros. }\end{array}$ \\
\hline Padrão Respiratório Ineficaz & $\begin{array}{l}\text { Inspiração e/ou expiração que não proporciona } \\
\text { ventilação adequada. }\end{array}$ & $\begin{array}{l}\text { Posição do corpo que inibe a expansão } \\
\text { pulmonar, ansiedade, dor, fadiga, entre } \\
\text { outros. }\end{array}$ \\
\hline Risco de Débito Cardíaco diminuído & $\begin{array}{l}\text { Suscetibilidade a volume de sangue bombeado pelo } \\
\text { coração inadequado para atender às demandas } \\
\text { metabólicas do organismo que pode comprometer a } \\
\text { saúde. }\end{array}$ & $\begin{array}{l}\text { Alteração na contratilidade, Alteração } \\
\text { na frequência cardíaca, Alteraçãa na } \\
\text { pós-carga, Alteração no risco cardíaco, } \\
\text { entre outros. }\end{array}$ \\
\hline Risco de Hipotermia & $\begin{array}{l}\text { Suscetibilidade a falha na termorregulação que pode } \\
\text { resultar em temperatura corporal central abaixo dos } \\
\text { parâmetros diurnos normais, que pode comprometer } \\
\text { a saúde. }\end{array}$ & $\begin{array}{l}\text { Dano ao hipotálamo, Controle vascular } \\
\text { ineficaz, trauma, entre outros. }\end{array}$ \\
\hline Dor no trabalho de parto & $\begin{array}{l}\text { Experiência sensorial e emocional, que varia de } \\
\text { agradável a desagradável, associada ao trabalho de } \\
\text { parto e nascimento da criança. }\end{array}$ & Dilatação cervical e expulsão do feto. \\
\hline
\end{tabular}

O trauma acarreta consequências sociais e econômicas tanto para os indivíduos quanto para a sociedade. As lesões relacionadas a ele podem ocasionar incapacidades físicas e ou mentais, temporárias ou permanentes e também levar ao óbito ${ }^{(7)}$.
Para a identificação das respostas do indivíduo ao trauma, o enfermeiro deve possuir uma base sólida em semiologia, anatomia e fisiopatologia, agregando sua experiência clínica e de suas habilidades de interação e observação.
Entende-se assim, que esse conjunto de habilidades é essencial para o julgamento clínico e consequente tomada de decisão. Os cuidados pré-hospitalares podem fazer a diferença entre a vida e a morte, entre uma sequela temporária, 
grave, ou permanente, ou ainda, entre uma vida produtiva e uma destituída de bem-estar. As intervenções que auxiliam o enfermeiro neste cenário do APH estão embasadas em diretrizes preconizadas universalmente ${ }^{(17)}$.

Com relação às parturientes, os resultados mostraram que os diagnósticos de enfermagem expressam os fenômenos intrínsecos às necessidades das mulheres que vivenciam a parturição e representam elementos que devem ser o foco da atuação do enfermeiro no trabalho de parto e no parto. São apresentados como reais e de risco e estão relacionados aos aspectos funcionais, emocionais e ambientais de acordo com as necessidades humanas básicas, segundo o modelo assistencial de Horta. Assim, a respectiva elaboração exigiu a utilização da NANDA-I como sistema de classificação, a qual permite a padronização da linguagem profissional para determinação dos conceitos identificados na prática assistencial ${ }^{(18)}$.

Verificou-se que os diagnósticos de enfermagem com suas características definidoras, acompanhados pelas intervenções de enfermagem, estavam diretamente relacionadas com às gestantes dentro do trauma.

É importante ressaltar que quando se está diante de uma situação no ambiente de serviço de atendimento móvel de urgência, o enfermeiro como profissional de saúde precisa ter conhecimento para saber prestar os devidos cuidados necessários às gestantes como oxigenação/respiração e circulação, prevenindo qualquer alteração grave do quadro, mantendo sempre que possível a vitalidade da mãe e do bebê.

Aponta-se que a oferta inadequada de sangue oxigenado ao cérebro e a outras estruturas vitais é o fator que mais rapidamente causa a morte do traumatizado. A prevenção da hipoxemia requer uma via aérea protegida e desobstruída e uma ventilação adequada. É imperativo assegurar uma via aérea permeável, administrar oxigênio e proporcionar suporte ventilatório(17).

Ressalta-se também que a compres-

são direta aplicada sobre o local de sangramento é a técnica inicial empregada para o controle de hemorragia externa, aumentando a pressão extraluminal e, portanto, reduzindo a pressão transluminal, ajudando a diminuir ou parar o sangramento. Mesmo se a perda sanguínea não for completamente interrompida, ela pode diminuir até o ponto em que o sistema de coagulação do sangue possa parar a hemorragia, dependendo de cada caso ${ }^{(19)}$.

A prevenção de lesões exige, da equipe, a aplicação de estratégias passivas e ativas capazes de proporcionar proteção automática durante o atendimento, o uso do colar cervical, protetor lateral de cabeça, prancha rígida, dentre outros equipamentos e materiais que reduzem os riscos de trauma e lesão(17).

Com isso, no atendimento pré-hospitalar, a ameaça pode ser a doença preexistente, o tipo de trauma ocorrido, a falta de segurança no local da cena do acidente ou a própria exposição ambiental, portanto, a segurança deve ser prevista tanto para a equipe do $\mathrm{APH}$, como para o paciente. Nesses casos, a equipe do APH deverá remover o paciente imediatamente para um ambiente seguro antes de iniciar a avaliação e as intervenções necessárias ${ }^{(17)}$.

\section{CONCLUSÃO}

cientificidade à

prática profissional.

Portanto entende-

se, que este

processo deve ser

adotado em todos

os atendimentos

deste cenário.
Sendo assim, como estabelecido inicialmente, este estudo identificou diagnósticos de enfermagem no trauma em gestante, para proporcionar uma melhor assistência no ambiente pré-hospitalar. Ressaltando que é uma ação privativa do enfermeiro, pois os profissionais da área da saúde devem ser qualificados, tanto tecnicamente quanto cientificamente, para o estabelecimento destes diagnósticos.

Compreende-se, também, que a realização da assistência de enfermagem precisa ser qualificada e benéfica a estas mulheres no período-gravídico, encontradas no serviço de atendimento móvel de urgência, que atribui melhor e com maior cientificidade à prática profissional. Portanto entende-se, que este processo deve ser adotado 
em todos os atendimentos deste cenário.

Conclui-se, como contribuições do estudo para o avanço da prática profissional e do conhecimento científico da enfermagem que, com os diagnósticos de enfermagem no trauma em gestante estabelecidos, inicia uma seleção de intervenções de enfermagem de qualidade para diminuir a morbimortalidade deste cenário.

\section{REFERÊNCIAS}

1. Conselho Nacional de Saúde, Ministério da Saúde (BR). Portaria n. ${ }^{\circ}$ 316/04 - Qualifica o Serviço de Atendimento Móvel às Urgências - SAMU 192, no município de Maceió (AL), à Rede Nacional de Atenção às Urgências. Brasília (Brasil): Ministério da Saúde; 2004.

2. Ministério da Saúde (BR). Portaria n. 1.864, de 29 de setembro de 2003. Institui o componente pré-hospitalar móvel da Política Nacional de Atenção às Urgências. Diário Oficial da União (Brasília). 2003 Out 5.

3. Ministério da Saúde (BR). Política nacional de atenção às urgências [Internet]. 3rd ed. Brasília: Ministério da Saúde; 2006 [cited 2018 Apr 17]. Available from: http://bvsms.saude.gov.br/bvs/publicacoes/politica_nacional_atencao_urgencias_3ed.pdf.

4. Ministério da Saúde, Secretaria de Atenção à Saúde, Departamento de Atenção Especializada (BR). Manual instrutivo da rede de atenção às urgências e emergências no Sistema Único de Saúde (SUS) [Internet]. Brasília: Ministério da Saúde; 2013 [cited 2018 Mar 18]. Available from: http:// bvsms.saude.gov.br/bvs/publicacoes/ manual_instrutivo_ rede_atencao_urgencias.pdf.

5. Almeida MS, Silva IA. Necessidades de mulheres no puerpério imediato em uma maternidade pública de Salvador, Bahia, Brasil. Rev Esc Enferm USP [Internet]. 2008 [citado 2014 nov 12]; 42(2):347-54. Disponivel em: http://www. scielo.br/pdf/reeusp/v42n2/a18.pdf.

6. Sallum AMC, Santos JFL, Lima FD. Nursing diagnoses in trauma victims with fatal outcomes in the emergency scenario. Rev Lat Am Enfermagem [Internet]. 2012 [acesso em: 20 dez 2013]; 20(1):3-10. DOl: http://dx.doi.org/10.1590/ S0104-11692012000100002.

7. Cyrillo RMZ, Dalri MCB, Canini SRMS, Carvalho EC, Lourencini RR. Diagnósticos de enfermagem em vítimas de trauma atendidas em um serviço pré-hospitalar avançado móvel. Rev. Eletr. Enf. [Internet]. 2009 [cited 2011 out 15]; 11(4):811-9. Available from: http://www.fen.ufg.br/revista/ v11/n4/pdf/v11n4a06.pdf.

8. Galdeano LE, Rossi LA. Construção e validação de instrumento de coleta de dados para o período perioperatório de cirurgia cardíaca. Rev. Latino-Am. Enfermagem. 2002; 10(6):800-4.

9. Carvalho EC. Relações entre a coleta de dados, diagnóstico e prescrição de enfermagem a pacientes adultos de uma UTI. Rev. Latino-Am. Enfermagem. 2008; 16(4):700-6.

10. Silva MRC, Vieira BDG, Alves VH, Rodrigues DP, Vargas GSA, Sá AMP. A percepção de gestantes de alto risco acerca do processo de hospitalização. Rev Enferm UER] [Inter- net]. 2013 [citado 2014 nov 10];21(2):792-7. Disponível em: http://www.facenf.uerj.br/v21esp2/v21e2a16.pdf.

11. Conselho Federal de Enfermagem (COFEN). Resolução n. ${ }^{\circ}$ 358/2009, de 15 de outubro de 2009. Dispõe sobre a Sistematização da Assistência de Enfermagem e a implementação do Processo de Enfermagem em ambientes, públicos ou privados, em que ocorre o cuidado profissional de Enfermagem, e dá outras providências [Internet]. COFEN Legislação e Normas. 2009 [cited 2012 May 30]. Disponível em: http://site.portalcofen.gov.br/resolucao/2009.

12. Amante LN, Rossetto AP, Schneider DG. Sistematização da assistência de enfermagem em unidade de terapia intensiva sustentada pela teoria de Wanda Horta. Rev. esc. enferm. USP [Internet]. 2009 [cited 2011 maio 26]; 43(1):54-64. Available from: http://www.scielo.br/scielo.php?script=sci_ arttext $\&$ pid $=$ S0080 $=62342009000100007-\&$ Ing=pt\&nrmiso.

13. Caritá EC, Nini RA, Melo AS. Sistema de auxílio aos diagnósticos de enfermagem para vítimas de trauma no atendimento avançado pré-hospitalar móvel utilizando as Taxonomias NANDA e NIC. J. Health Inform. 2010 Out-Dez; 2(4):87-94.

14. Martins I, Gutiérrez MGR. Intervenções de enfermagem para o diagnóstico de enfermagem desobstrução ineficaz de vias aéreas. Acta Paul Enferm. 2005; 18(2):143-9.

15. Rosenthal GE, Halloran EJ, Kiley M, Pinkley C, Landefeld CS. Development and validation of the Nursing Severity Index. A new method for measuring severity of illness using diagnoses. Med Care. 1992; 30(12):1127-41.

16. Herdman, T. Heather; Kamitsuru, Shigemi. Diagnósticos de enfermagem da NANDA - I: Definições e classificação. Edição $11^{\circ}$. Artmed Editora Ltda; 2018.

17. National Association of Emergency Medical Technicians (U.S.). PHTLS basic and advanced Prehospital Trauma Life Support. 6. ed. St Louis: Mosby; 2007.

18. Marin HF, Barbieri M, Barros SMO. Conjunto internacional de dados essenciais de enfermagem: comparação com dados na área de saúde da mulher. Acta Paul Enferm [Internet]. 2010 [citado 2014 out 02]; 23(2):251-6. Disponivel em: http://www.scielo.br/pdf/ape/v23n2/16.pdf.

19. Coutinho APO. Construção e validação de um instrumento para a sistematização da assistência de enfermagem no Serviço de Atendimento Móvel de Urgência (SAMU) [dissertação]. João Pessoa: Universidade Federal da Paraíba/UFP [Internet]; 2007. 124 p. [cited 2011 mai 02]. Available from: http://www.dominiopublico.gov.br/pesquisa/DetalheObraForm.do?select_action=\&co_obra $=60030$. 